\title{
Does treatment guided by exhaled nitric oxide fraction improve outcomes in subgroups of children with asthma?
}

\author{
Shona S. Fielding ${ }^{1}$, Marielle Pijnenburg ${ }^{2}$, Johan de Jongste ${ }^{2}$, Katherine Pike ${ }^{3,4}$, \\ Graham Roberts ${ }^{3}$, Helen Petsky ${ }^{5}$, Anne B. Chang ${ }^{6,7}$, Maria Fritsch ${ }^{8}$, \\ Thomas Frischer $^{8}$, Stanley J. Szefler ${ }^{9}$, Peter Gergen ${ }^{10}$, Francoise Vermeulen ${ }^{11}$, \\ Robin Vael ${ }^{12}$ and Steve S. Turner (1) ${ }^{13}$
}

Affiliations: ${ }^{1}$ Medical Statistics Team, Institute of Applied Health Sciences, University of Aberdeen, Aberdeen, UK. 'Dept of Paediatric Respiratory Medicine and Allergology, Erasmus MC - Sophia Children's Hospital, Rotterdam, The Netherlands. ${ }^{3}$ Clinical and Experimental Science Academic Unit, University of Southampton, Southampton, UK. ${ }^{4}$ Respiratory Critical Care and Anaesthesia Group, Institute of Child Health, University College London, London, UK. ${ }^{5}$ School of Nursing and Midwifery, Griffith University, Menzies Health Institute Queensland, Brisbane, Australia. ${ }^{6}$ Dept of Respiratory and Sleep Medicine, Queensland Children's Hospital, Queensland University of Technology, Brisbane, Australia. ${ }^{7}$ Child Health Division, Menzies School of Health Research, Charles Darwin University, Darwin, Australia. ${ }^{8}$ University Children's Hospital, Vienna, Austria. ${ }^{9}$ Breathing Institute, Children's Hospital Colorado, Dept of Pediatrics, University of Colorado School of Medicine, Aurora, CO, USA. ${ }^{10}$ National Institute of Allergy and Infectious Diseases, Bethesda, MD, USA. ${ }^{11}$ Pediatric Dept, Hôpital Erasme, Université Libre de Bruxelles (U.L.B.), Brussels, Belgium. ${ }^{12}$ Dept of Paediatrics, Antwerp University Hospital, Antwerp, Belgium. ${ }^{13}$ Child Health, University of Aberdeen, Aberdeen, UK.

Correspondence: Steve Turner, Child Health, Royal Aberdeen Children's Hospital, Aberdeen, AB25 2ZG, UK. E-mail: s.w.turnerdabdn.ac.uk

@ERSpublications

Some children with asthma may have better outcomes than others if their treatment is guided by exhaled nitric oxide http://bit.ly/2VahxJV

Cite this article as: Fielding SS, Pijnenburg M, de Jongste J, et al. Does treatment guided by exhaled nitric oxide fraction improve outcomes in subgroups of children with asthma? Eur Respir J 2020; 55: 1901879 [https://doi.org/10.1183/13993003.01879-2019].

\section{ABSTRACT}

Introduction: Exhaled nitric oxide fraction $\left(F_{\mathrm{ENO}}\right)$, a biomarker of eosinophilic airway inflammation, may be useful to guide asthma treatment. $F_{\mathrm{ENO}}$-guided treatment may be more effective in certain subgroups for improving asthma outcomes compared to standard treatment.

Methods: An individual patient data analysis was performed using data from seven randomised clinical trials (RCTs) which used $F_{\mathrm{ENO}}$ to guide asthma treatment. The incidence of an asthma exacerbation and loss of control, and the time to first exacerbation and loss of control were described between five subgroups of RCT participants.

Results: Data were available in 1112 RCT participants. Among those not treated with leukotriene receptor antagonists (LTRA), but not among those who were treated with LTRA, $F_{\text {ENO-guided treatment was }}$ associated with reduced exacerbation risk (OR 0.68, 95\% CI 0.49-0.94), longer time to first exacerbation (hazard ratio (HR) $0.76,95 \%$ CI $0.57-0.99$ ) and borderline reduced risk for loss of control (OR 0.70, 95\% CI 0.49-1.00). Nonobese children, compared to obese children, were less likely to lose asthma control when treatment was guided by $F_{\mathrm{ENO}}(\mathrm{OR} 0.69,95 \%$ CI $0.48-0.99$ ) and time to loss of control was longer (HR 0.77, 95\% CI 0.61-0.99).

Conclusions: Asthma treatment guided by $F_{\mathrm{ENO}}$ may be more effective in achieving better asthma outcomes for patients who are not treated with LTRA and who are not obese, compared to standard practice. 


\section{Introduction}

Asthma is a common chronic condition which affects 1 million children in the UK [1], 6 million children in the US [2] and 235 million children and adults around the world [3]. There is effective treatment to control asthma symptoms and guidelines recommend that treatment should be titrated to asthma symptoms [4-6]. There remains a widely accepted recognition that an objective measurement to guide asthma treatment is required [7].

Exhaled nitric oxide $\left(F_{\mathrm{ENO}}\right)$ has many of the characteristics required of an objective tool to measure asthma symptoms. For example, $F_{\mathrm{ENO}}$ rises before symptoms occur $[8,9]$, falls when asthma treatment is administered $[10,11]$, can be measured with minimal discomfort to the patient and results are available within a few minutes using commercially available apparatus [12]. A meta-analysis including eight clinical trials in children and young adults found that addition of $F_{\mathrm{ENO}}$ measurements to symptom-guided treatment did not reduce asthma symptoms [13], but that $F_{\mathrm{ENO}}$ guided treatment reduced asthma exacerbations [13].

Asthma is a heterogeneous condition and what we do not know is whether there are patient subgroups in whom using $F_{\mathrm{ENO}}$ to guide asthma treatment may be beneficial [7]. In one randomised controlled trial (RCT), the intervention was more effective in participants who had more positive skin tests and who were obese, but age, sex, asthma severity and initial $F_{\text {ENO }}$ concentration were not associated with a different outcome from the intervention [14]. In a second RCT there was no evidence of improved outcomes between individuals who were concordant or discordant for $F_{\mathrm{ENO}}$ and symptoms [15].

Our group has pooled the data collected from seven of the eight published RCTs where the efficacy of $F_{\mathrm{ENO}}$ used to guide asthma treatment was examined, compared to standard management [16]. Here we use data from 1112 participants to test the hypothesis that there are particular subgroups of patients where $F_{\mathrm{ENO}}$-guided treatment is more effective in improving asthma outcomes compared to standard treatment.

\section{Methods}

\section{Study design}

Authors of all published RCTs where measurements of $F_{\mathrm{ENO}}$ were used to guide asthma treatment in children [17] were contacted and asked to provide data, as described previously [16]. The children who took part in the studies were recruited from hospital clinics and were followed-up for between 6 and 12 months. The primary outcome was the presence of any asthma exacerbation during follow-up [13]. Secondary outcomes were loss of control among those who were initially controlled and time to first exacerbation and time to first loss of control. Institutional ethical approval was provided for each trial which contributed data.

\section{Details of each population}

FRITSCH et al. [18] undertook a study of 47 children with asthma attending a hospital asthma clinic in Vienna (Austria) and collected data (including $F_{\mathrm{ENO}}$, asthma symptom score and history of recent exacerbations) at 6-week intervals over 6 months. PEIRSMAn et al. [19] recruited 99 participants with persistent asthma attending hospital asthma clinics across Belgium and collected data at 3-month intervals over 12 months. Petsky et al. [20] recruited 63 children from hospital clinics in Australia and Hong Kong, and data were collected on eight occasions over 12 months (at 1, 2, 3, 4, 6, 8, 10 and 12 months). Pijnenburg et al. [21] included 86 participants attending a single hospital clinic in the Netherlands and data were collected at baseline, 3, 6, 9 and 12 months. PIKE et al. [22] recruited 90 participants in clinics in four UK hospitals and collected data at 2-month intervals over a year. SzEFLER et al. [14] recruited 546 participants from the community in the USA and collected post-randomisation information over 46 weeks including at 3 months, 6 months, 8 months and 10 months. VOOREND-VAN BERGEN et al. [23] undertook a study of 181 participants attending hospital clinics in the Netherlands and collected data at 4-month intervals over a year. The treatment algorithms in $F_{\mathrm{ENO}}$-guided and standard-practice arms in each RCT were different to other RCTs (table 1).

\section{Data collected}

Covariates collected at baseline in all trials included age, sex, height, weight, treatment arm, dose of inhaled corticosteroid (ICS, as daily budesonide equivalent dose), prescribed long-acting $\beta$-agonist (LABA) or not, prescribed leukotriene receptor agonist (LTRA) or not, and an asthma control score. Ethnicity was available in four cohorts [14, 21-23]. Body mass index (BMI) was derived and International Obesity Task Force weight categories created [28]; obesity is defined as equivalent to adult BMI $\geqslant 30 \mathrm{~kg} \cdot \mathrm{m}^{-2}$. Forced expiratory volume in $1 \mathrm{~s}\left(\mathrm{FEV}_{1}\right)$ (\% predicted) was calculated according to the Global Lung Initiative standard [29], apart from in two trials [21, 22] in which only percentage $\mathrm{FEV}_{1}$ standardised to other references was available. $F_{\mathrm{ENO}}$ was measured in all studies in accordance with the 2005 guideline [30]. At 
TABLE 1 A summary of characteristics of the randomised controlled trials whose data were used for the present analysis

\begin{tabular}{|c|c|c|c|c|c|c|c|}
\hline \multirow{2}{*}{$\begin{array}{l}\text { First author, } \\
\text { year [ref.] } \\
\text { country }\end{array}$} & \multirow{2}{*}{$\begin{array}{l}\text { Age } \\
\text { years }\end{array}$} & \multirow{2}{*}{$\begin{array}{l}\text { Inclusion criteria } \\
\text { (in addition to child } \\
\text { diagnosed with } \\
\text { asthma) }\end{array}$} & \multirow{2}{*}{$\begin{array}{c}\text { Definition of asthma } \\
\text { control }\end{array}$} & \multicolumn{2}{|c|}{ Treatment strategy } & \multirow{2}{*}{$\begin{array}{l}\text { Treatment options } \\
\text { (same for both } \\
\text { groups in all } \\
\text { studies) }\end{array}$} & \multirow{2}{*}{$\begin{array}{l}\text { What did the trial find? } \\
\text { ( } F_{\mathrm{ENO}} \text { treatment } \\
\text { compared to standard } \\
\text { care })\end{array}$} \\
\hline & & & & Intervention group & Control group & & \\
\hline $\begin{array}{l}\text { FRITSCH, } 2006 \\
\text { [18] Austria }\end{array}$ & $11.5 \pm 3.1$ & $\begin{array}{l}\text { Age 6-18 years } \\
\text { Sensitised to inhaled } \\
\text { allergens } \\
\text { No systemic } \\
\text { corticosteroids } \\
1 \text { month before } \\
\text { recruitment }\end{array}$ & $\begin{array}{c}\mathrm{FEV}_{1}>80 \% \text { pred, } \\
<6 \text { doses of SABA over } \\
14 \text { days and no or mild } \\
\text { symptoms }\end{array}$ & $\begin{array}{l}\text { Step up if control lost } \\
\text { regardless of } F_{\text {ENO }} \\
\text { Step down if controlled } \\
\text { and } F_{\text {ENO }} \leqslant 20 \mathrm{ppb} \\
\text { If } F_{\text {ENO }}>20 \mathrm{ppb} \text { and } \\
\text { controlled, step up, unless } \\
\text { already on ICS (in which } \\
\text { case no change) }\end{array}$ & $\begin{array}{l}\text { Step up if either FEV } \\
<80 \% \text { pred or } \\
\text { symptoms severe or } \\
\geqslant 6 \text { doses of SABA } \\
\text { over previous } 14 \text { days } \\
\text { Step down if } \\
\text { controlled }\end{array}$ & Four treatment steps & $\begin{array}{l}\text { Higher mid-expiratory } \\
\text { flow, higher dose of ICS }\end{array}$ \\
\hline $\begin{array}{l}\text { Peirsman, } 2014 \\
\text { [19] Belgium }\end{array}$ & $10.7 \pm 2.1$ & $\begin{array}{l}\text { Sensitised to inhaled } \\
\text { allergens } \\
\text { No exacerbation or } \\
\text { systemic } \\
\text { corticosteroids } \\
3 \text { months before } \\
\text { recruitment }\end{array}$ & $\begin{array}{l}\text { A score of } \leqslant 3 \text { from the } \\
\text { first four lof seven) } \\
\text { questions on } A C T, F E V_{1} \\
>80 \% \text { and } \leqslant 2 \text { doses of } \\
\text { SABA over a week }\end{array}$ & $\begin{array}{l}\text { Step up if } F_{\text {ENO }}>20 \mathrm{ppb} \\
\text { regardless of control } \\
\text { Consider stepping up if } \\
F_{\text {ENO }} \leqslant 20 \mathrm{ppb} \text { and partly/ } \\
\text { fully uncontrolled } \\
\text { Step down if controlled } \\
\text { and } F_{\text {ENO }} \leqslant 20 \mathrm{ppb}\end{array}$ & $\begin{array}{c}\text { Step up if } \\
\text { uncontrolled, } \\
\text { consider stepping up } \\
\text { if partly controlled } \\
\text { Step down if } \\
\text { controlled }\end{array}$ & $\begin{array}{l}\text { Step up and down } \\
\text { options if on the } \\
\text { following preventers: } \\
\text { ICS alone; LTRA } \\
\text { alone; ICS + LABA; } \\
\text { ICS + LTRA }\end{array}$ & $\begin{array}{c}\text { Reduced exacerbations, } \\
\text { increased LTRA and } \\
\text { ICS dose } \\
\text { No difference in primary } \\
\text { outcome }\end{array}$ \\
\hline $\begin{array}{l}\text { PetSky, } 2015 \\
\text { [20] } \\
\text { Australia }\end{array}$ & $10.0 \pm 3.2$ & $\begin{array}{c}\text { Age }>4 \text { years } \\
\text { Prescribed asthma } \\
\text { preventer } \\
\text { Adherent to treatment }\end{array}$ & $\begin{array}{l}\text { Symptom score }{ }^{\#} \text { no } \\
\text { more than } 15 \% \text { higher } \\
\text { than previous } \\
\text { assessment (only used } \\
\text { for the control group) }\end{array}$ & $\begin{array}{l}\text { Step up or down based } \\
\text { only on } F_{\text {ENO }}>10 \text { or } \leqslant 10 \\
\text { ppb if nonatopic, }>12 \text { or } \\
\leqslant 12 \text { ppb with } 1 \text { positive } \\
\text { skin test, }>20 \text { or } \leqslant 20 \text { for }>1 \\
\text { positive skin test }\end{array}$ & $\begin{array}{l}\text { Step up if symptoms } \\
\text { score }>15 \% \text { higher } \\
\text { than previously } \\
\text { Step down if } \\
\text { symptoms score }<10\end{array}$ & $\begin{array}{l}\text { Seven steps (none } \\
\text { including LTRA) }\end{array}$ & $\begin{array}{l}\text { Reduced exacerbation, } \\
\text { increased ICS dose }\end{array}$ \\
\hline $\begin{array}{l}\text { PiJnenbuRG, } \\
2005[21] \\
\text { The } \\
\text { Netherlands }\end{array}$ & $12.3 \pm 2.8$ & $\begin{array}{l}\text { Age 6-18 years } \\
\text { Sensitised to inhaled } \\
\text { allergens } \\
\text { ICS dose unchanged } \\
\text { for } \geqslant 3 \text { months at } \\
\text { recruitment }\end{array}$ & $\begin{array}{c}\text { Score of }>14 \text { on } \\
\text { validated symptom } \\
\text { diary" }\end{array}$ & $\begin{array}{l}\text { Step up if } F_{\text {ENO }}>30 \mathrm{ppb} \\
\text { regardless of control } \\
\text { Treatment stepped down if } \\
\text { symptoms controlled and } \\
F_{\text {ENO }} \leqslant 30 \mathrm{ppb} \\
\text { No change if symptoms not } \\
\text { controlled but } F_{\mathrm{ENO}} \leqslant 30 \mathrm{ppb}\end{array}$ & $\begin{array}{l}\text { Step up if symptoms } \\
\text { uncontrolled } \\
\text { Step down if } \\
\text { controlled for second } \\
\text { assessment } \\
\text { No change if } \\
\text { controlled on first } \\
\text { assessment }\end{array}$ & $\begin{array}{c}\text { Nine steps (none } \\
\text { including LABA or } \\
\text { LTRA) }\end{array}$ & $\begin{array}{c}\text { Reduced } F_{\text {ENO }} \text { and } \\
\text { bronchial } \\
\text { hyperresponsiveness } \\
\text { No increase in ICS dose }\end{array}$ \\
\hline $\begin{array}{l}\text { PIKE, } 2013 \text { [22] } \\
\text { UK }\end{array}$ & $11.9 \pm 2.6$ & $\begin{array}{c}\text { Age } 6-17 \text { years } \\
\text { Prescribed } \geqslant 400 \mu \mathrm{g} \\
\text { ICS daily (budesonide } \\
\text { equivalent) } \\
\text { Adherent to treatment } \\
\text { No history of } \\
\text { life-threatening } \\
\text { asthma or requiring } \\
\text { maintenance oral } \\
\text { corticosteroids }\end{array}$ & $\begin{array}{l}\text { Modified validated } \\
\text { symptom diary }^{+}, \mathrm{FEV}_{1} \\
\geqslant 80 \% \text { and }<1 \text { SABA } \\
\text { dose per week }\end{array}$ & $\begin{array}{l}\text { Step up ICS if } F_{\text {ENO }} \geqslant 25 \mathrm{ppb} \\
\text { (or more than twice } \\
\text { baseline value) regardless } \\
\text { of control or FEV } \mathrm{F}_{1} \\
\text { Step up with LABA if poorly } \\
\text { controlled and } F_{\text {ENO }}<25 \mathrm{ppb} \\
\text { Step down if } F_{\text {ENO }} \leqslant 15 \mathrm{ppb} \\
\text { and controlled on two } \\
\text { consecutive assessments }\end{array}$ & $\begin{array}{l}\text { Step up if } \\
\text { uncontrolled } \\
\text { Step down if } \\
\text { controlled on two } \\
\text { consecutive } \\
\text { assessments }\end{array}$ & Eight treatment steps & $\begin{array}{l}\text { No differences in } \\
\text { outcomes }\end{array}$ \\
\hline
\end{tabular}




\begin{tabular}{|c|c|c|c|c|c|c|c|}
\hline \multirow{2}{*}{$\begin{array}{l}\text { First author, } \\
\text { year [ref.] } \\
\text { country }\end{array}$} & \multirow{2}{*}{$\begin{array}{l}\text { Age } \\
\text { years }\end{array}$} & \multirow{2}{*}{$\begin{array}{l}\text { Inclusion criteria } \\
\text { (in addition to child } \\
\text { diagnosed with } \\
\text { asthma) }\end{array}$} & \multirow{2}{*}{$\begin{array}{c}\text { Definition of asthma } \\
\text { control }\end{array}$} & \multicolumn{2}{|c|}{ Treatment strategy } & \multirow{2}{*}{$\begin{array}{l}\text { Treatment options } \\
\text { (same for both } \\
\text { groups in all } \\
\text { studies) }\end{array}$} & \multirow{2}{*}{$\begin{array}{l}\text { What did the trial find? } \\
\text { ( } F_{\mathrm{ENO}} \text { treatment } \\
\text { compared to standard } \\
\text { care })\end{array}$} \\
\hline & & & & Intervention group & Control group & & \\
\hline $\begin{array}{c}\text { SZEFLER, } 2008 \\
\text { [14] USA }\end{array}$ & $14.4 \pm 2.1$ & $\begin{array}{c}\text { Age } 12-20 \text { years } \\
\text { Living in community } \\
\text { where } \geqslant 20 \% \\
\text { households were } \\
\text { below poverty } \\
\text { threshold } \\
\text { Persistent or } \\
\text { uncontrolled asthma if } \\
\text { on long-term } \\
\text { preventer } \\
\text { Nonsmoker }\end{array}$ & $\begin{array}{c}\text { Four levels of control } \\
\text { depending on a } \\
\text { symptom score (ACT) } \\
\text { and a series of } F E V_{1} \text {, } \\
\text { cut offs (FEV } 1 \geqslant 80 \% \\
\text { pred, } 70-79 \% \text { pred or } \\
>70 \% \text { pred) }\end{array}$ & $\begin{array}{l}\text { Step up by one, two or } \\
\text { three treatment levels } \\
\text { depending on symptoms } \\
\text { score, } \mathrm{FEV}_{1} \text { and } F_{\text {ENO } 0-20,} \\
20.1-30,30.1-40 \text { or } \\
>40 \mathrm{ppb} \\
\text { Step down if controlled on } \\
\text { two consecutive } \\
\text { assessments and } F_{\text {ENO }} \\
\leqslant 20 \mathrm{ppb}\end{array}$ & $\begin{array}{l}\text { Step up by one, two } \\
\text { or three treatment } \\
\text { levels depending on } \\
\text { symptoms score and } \\
\text { FEV } \\
\text { Step down if } \\
\text { controlled on two } \\
\text { consecutive } \\
\text { assessments }\end{array}$ & $\begin{array}{l}\text { Seven treatment } \\
\text { steps (including } \\
\text { low-dose } \\
\text { theophylline) }\end{array}$ & $\begin{array}{l}\text { Reduced exacerbations, } \\
\text { increased ICS dose } \\
\text { No difference in primary } \\
\text { outcome }\end{array}$ \\
\hline $\begin{array}{l}\text { VoOREND-VAN } \\
\text { BERGEN, } 2010 \\
\text { [23] The } \\
\text { Netherlands }\end{array}$ & $10.2 \pm 3.0$ & $\begin{array}{c}\text { Age } 4-18 \text { years } \\
\text { Sensitised to inhaled } \\
\text { allergens } \\
>9 \% \text { bronchodilator } \\
\text { response } \\
\text { Prescribed ICS for } \\
\geqslant 3 \text { months } \\
\text { Nonsmoker } \\
\text { No history of multiple } \\
\text { ICU admissions for } \\
\text { asthma }\end{array}$ & ACT score $\geqslant 20$ & $\begin{array}{c}\text { Step up if controlled and } \\
F_{\text {ENO }} \geqslant 50 \mathrm{ppb} \text { or } \\
\text { uncontrolled and } F_{\text {ENO }} \\
\geqslant 25 \mathrm{ppb} \\
\text { Step down if controlled } \\
\text { and } F_{\text {ENO }}<25 \mathrm{ppb} \\
\text { Otherwise no change }\end{array}$ & $\begin{array}{c}\text { Step up if } \\
\text { uncontrolled } \\
\text { Step down/no change } \\
\text { if controlled }\end{array}$ & $\begin{array}{l}\text { Seven treatment } \\
\text { steps }\end{array}$ & $\begin{array}{c}\text { Increased asthma control } \\
\text { No difference in } \\
\text { outcomes }\end{array}$ \\
\hline
\end{tabular}


each follow-up visit an assessment of asthma control was made (table 1) and history of any asthma attack since the previous assessment was recorded (defined as receipt of oral corticosteroids for an asthma exacerbation [16]). The trials used different symptom score methodology and loss of control was defined as per trial protocol by reaching a pre-agreed symptom score.

\section{Analysis}

Asthma outcomes were compared between participants in the $F_{\mathrm{ENO}}$-guided and standard treatment arms of RCTs for the following five subgroups defined at baseline and previously associated with differences in $F_{\text {ENO }}$. The five subgroups were stratified by dose of ICS $(\leqslant 400 \mu \mathrm{g}$ budesonide equivalent or $>400 \mu \mathrm{g})[10]$, use of LTRA [31], obesity [14], ethnicity (white versus other) [32] and atopic (i.e. positive skin-prick test or positive type-specific IgE) [14]. Any exacerbation during follow-up and time to first exacerbation and any loss of control and time to loss of control were calculated (the latter restricted to those who were controlled at baseline). Time to first exacerbation or to loss of control was determined using data collected at the scheduled study assessments, and supplementary table S1 describes the time in weeks between baseline and each follow-up assessment in each RCT. For example, if a participant experienced an exacerbation after their 3-month assessment but before the 6-month assessment, time was censored at 6 months. Logistic regression was used to relate any exacerbation or any loss of control to an interaction term between each baseline characteristic and treatment arm; a significant interaction term $(\mathrm{p}<0.05)$ would indicate that outcomes were different between $F_{\mathrm{ENO}}$-guided and standard treatment for a subgroup. Cox proportional hazards models were used to investigate time to first exacerbation or time to first loss of control. Each subgroup was considered separately and all models included adjustment for covariates associated with the outcome, including age, a variable for each RCT and ICS dose at baseline (this was not included in the ICS-dose subgroup model). Standard statistical software was used (Stata version 14; StataCorp, College Station, TX, USA) and significance was assumed at 5\%. All analyses were exploratory, so no adjustment was made for multiple comparisons.

\section{Results}

Study subjects

Data from seven RCTs were analysed [14, 18-23], totalling 1112 participants. Characteristics of participants at baseline have been described previously [16] and are presented in table 2. The majority of participants (58\%) were male and the mean \pm SD age was $12.6 \pm 3.1$ years. Characteristics of participants in the five subgroups are presented in supplementary table S2, i.e. LTRA treatment (yes/no), ICS dose $\leqslant 400 \mu \mathrm{g} />400 \mu \mathrm{g}$ ), obese (yes/no), atopic (yes/no) and white versus other ethnic group.

\section{$\mathrm{F}_{\text {ENO }}$ intervention and asthma exacerbation outcomes}

Any exacerbation

Of the 1047 participants for whom exacerbation data were available, 296 (28\%) had at least one exacerbation, with the first occurring after a median (interquartile range (IQR)) 22 (14-38) weeks. Table 3 shows the effect of treatment group was different for the two LTRA subgroups (interaction p-value 0.039). Those not treated with LTRA had lower odds for one or more exacerbations in the $F_{\mathrm{ENO}}$-guided group compared to standard care (OR 0.68, 95\% CI 0.49-0.94), but there was no difference observed between $F_{\mathrm{ENO}}$-guided and control groups for those on LTRA (table 3) The number needed to treat with

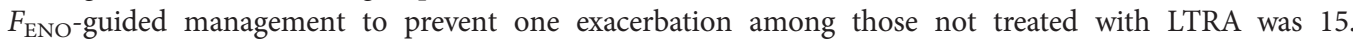
Interactions between treatment arm and other baseline characteristics (ICS dose, obesity, atopy and white ethnicity) were not significant when predicting exacerbation (table 3 ).

Time to first exacerbation

Overall, the median (IQR) time to first exacerbation was 22 (14-38) weeks in the standard arm and 22 (13-34) weeks in the $F_{\mathrm{ENO}}$-guided arm. The interaction term between treatment arm and LTRA was of borderline significance for time for first exacerbation $(\mathrm{p}=0.049)$, and among those not treated with LTRA at baseline, the time to first asthma exacerbation was slightly longer for participants receiving $F_{\mathrm{ENO}}$-guided treatment compared to standard care (hazard ratio (HR) $0.76,95 \%$ CI $0.57-0.99 ; \mathrm{p}=0.048$ ) (table 4 and figure 1). Time to first exacerbation was no different between treatment groups for those treated with LTRA. The interaction terms with treatment arm were not significant for ICS dose, atopy, obesity or ethnicity (table 4).

\section{$\mathrm{F}_{\text {ENO }}$ intervention and asthma control outcomes}

Any loss of asthma control

There were 787 participants who were controlled at baseline; 336 (43\%) remained controlled until completion of the trial, 344 (44\%) lost control and 107 (14\%) were lost to follow-up for this outcome. The median (IQR) time to loss of control in these 344 patients was 22 (13-30) weeks. There was no difference 


\begin{tabular}{|c|c|c|c|c|c|c|c|c|}
\hline & $\begin{array}{c}\text { FRITSCH } \\
{[18]}\end{array}$ & $\begin{array}{l}\text { Peirsman } \\
\text { [19] }\end{array}$ & $\begin{array}{l}\text { PetsKY } \\
{[20]}\end{array}$ & $\begin{array}{l}\text { PiJnenburg } \\
\text { [21] }\end{array}$ & $\begin{array}{l}\text { PIKE } \\
\text { [22] }\end{array}$ & $\begin{array}{l}\text { SZEFLER } \\
{[14]}\end{array}$ & $\begin{array}{c}\text { VOOREND-VAN BERGEN } \\
\text { [23] }\end{array}$ & $\begin{array}{l}\text { All populations } \\
\text { combined }\end{array}$ \\
\hline Participants n & 47 & 99 & 63 & 86 & 90 & 546 & 181 & 1112 \\
\hline Male $\%$ (n) & $60(28)$ & $67(66)$ & $49(31)$ & $65(56)$ & $57(51)$ & 53 (288) & 68 (123) & $58(643)$ \\
\hline Age years & $11.5 \pm 3.1$ & $10.7 \pm 2.1$ & $10.0 \pm 3.2$ & $12.3 \pm 2.8$ & $10.9 \pm 2.6$ & $14.4 \pm 2.1$ & $10.2 \pm 3.0$ & $12.6 \pm 3.1$ \\
\hline$F_{\text {ENO }} \mathrm{ppb}$ & $\begin{array}{c}34(18.6-58.6) \\
n=46\end{array}$ & $\begin{array}{c}31(14-69) \\
n=49\end{array}$ & $\begin{array}{c}26(12.2-47.5) \\
n=61\end{array}$ & $\begin{array}{c}32(16.6-52.5) \\
n=86\end{array}$ & $\begin{array}{c}26(10-48) \\
n=90\end{array}$ & $\begin{array}{c}20(11.2-40.6) \\
n=546\end{array}$ & $\begin{array}{c}18(10.2-30.4) \\
n=179\end{array}$ & $\begin{array}{c}22(11.6-43.0) \\
n=1057\end{array}$ \\
\hline $\mathrm{FEV}_{1} \%$ pred & $\begin{array}{c}93.5 \pm 15.7 \\
n=47\end{array}$ & $\begin{array}{c}91.4 \pm 15.7 \\
n=98\end{array}$ & $\begin{array}{c}90.7 \pm 15.6 \\
n=54\end{array}$ & $\begin{array}{c}97.5 \pm 17.5 \\
n=86\end{array}$ & $\begin{array}{c}89.2 \pm 14.3 \\
n=90\end{array}$ & $\begin{array}{c}90.9 \pm 16.6 \\
n=546\end{array}$ & $\begin{array}{c}93.8 \pm 13.0 \\
n=157\end{array}$ & $\begin{array}{c}93.5 \pm 18.1 \\
n=1078\end{array}$ \\
\hline Atopic & 100 & 100 & 38 (24/63) & 100 & 76 (68/90) & 88 (467/531) & 100 & 89 (972/1097) \\
\hline Obese & 8 (4/47) & $1(1 / 99)$ & $2(1 / 58)$ & $4(4 / 85)$ & 8 (7/89) & $31(165 / 526)$ & $3(5 / 181)$ & $17(187 / 1085)$ \\
\hline LTRA & $28(13 / 47)$ & 60 (59/99) & $10(6 / 58)$ & $0(0 / 86)$ & $51(46 / 90)$ & $15(80 / 546)$ & $13(23 / 181)$ & $21(227 / 1107)$ \\
\hline LABA & $38(18 / 47)$ & 32 (32/99) & $67(39 / 58)$ & $38(33 / 86)$ & 76 (68/90) & $66(360 / 546)$ & $46(84 / 181)$ & $57(634 / 1107)$ \\
\hline ICS dose $\mu \mathrm{g}$ & $400(0-800)$ & $320(200-400)$ & $400(250-500)$ & $800(400-1000)$ & $800(400-1000)$ & $1000(400-2000)$ & $400(400-800)$ & $400(400-1000)$ \\
\hline$>400 \mu \mathrm{g}$ BUD & $30(14 / 47)$ & $15(15 / 99)$ & $49(31 / 63)$ & $66(57 / 86)$ & $59(53 / 90)$ & $53(287 / 546)$ & 33 (59/181) & $46(516 / 1112)$ \\
\hline $\begin{array}{l}\text { White ethnic } \\
\text { group }\end{array}$ & Not stated & 82 (69/84) & Not stated & Not stated & 92 (83/90) & $0(0 / 526)$ & $89(160 / 179)$ & 35 (312/901) \\
\hline \multicolumn{9}{|l|}{ Control status } \\
\hline Controlled & 49 (23/47) & 75 (49/65) & $72(41 / 57)$ & $57(44 / 77)$ & 97 (87/90) & $80(421 / 528)$ & $67(122 / 181)$ & 75 (787/1045) \\
\hline Not controlled & $51(24 / 47)$ & $25(16 / 65)$ & $28(16 / 57)$ & $43(33 / 77)$ & 3 (3/90) & $20(107 / 528)$ & $33(59 / 181)$ & $24(258 / 1045)$ \\
\hline
\end{tabular}

Data are presented as mean $\pm \mathrm{SD}$, median (interquartile range) or $\%(\mathrm{n} / \mathrm{N})$, unless otherwise stated. $F_{\mathrm{ENO}}$ : exhaled nitric oxide fraction; $\mathrm{FEV}_{1}$ : forced expiratory volume in $1 \mathrm{~s} ; \%$ pred: $\%$ predicted; LTRA: leukotriene receptor antagonist; LABA: long-acting $\beta$-agonist; ICS: inhaled corticosteroid; BUD: budesonide-equivalent ICS. 
TABLE 3 Proportion of individuals with any asthma exacerbation in exhaled nitric oxide fraction $\left(F_{\text {ENO }}\right)$-guided and standard management arms of clinical trials with stratification for baseline patient characteristics

\begin{tabular}{|c|c|c|c|c|}
\hline & \multicolumn{2}{|c|}{$\geqslant 1$ exacerbation in each treatment arm } & \multirow[t]{2}{*}{$\begin{array}{c}F_{\text {ENo }} \text { versus } \\
\text { standard }\end{array}$} & \multirow[t]{2}{*}{$\begin{array}{l}p \text {-value for } \\
\text { interaction" }\end{array}$} \\
\hline & $\begin{array}{l}F_{\mathrm{ENO}}-\text { guided } \\
\text { management }\end{array}$ & $\begin{array}{c}\text { Standard } \\
\text { management }\end{array}$ & & \\
\hline \multicolumn{5}{|l|}{ LTRA treatment } \\
\hline Yes & 49/109 (45) & 40/104 (38) & $1.46(0.76-2.79)$ & \multirow{2}{*}{0.039} \\
\hline No & $88 / 410(21)$ & $119 / 419(28)$ & $0.68(0.49-0.94)$ & \\
\hline \multicolumn{5}{|l|}{ ICS dose } \\
\hline$\leqslant 400 \mu \mathrm{g}$ BUD & 48/289 (17) & $58 / 279(21)$ & $0.72(0.46-1.11)$ & \multirow[t]{2}{*}{0.493} \\
\hline$>400 \mu \mathrm{g}$ BUD & $89 / 232(38)$ & $101 / 247(41)$ & $0.88(0.60-1.28)$ & \\
\hline \multicolumn{5}{|l|}{ Obese } \\
\hline Yes & $30 / 88(34)$ & $36 / 81(44)$ & $0.63(0.33-1.21)$ & \multirow[t]{2}{*}{0.342} \\
\hline No & $107 / 425(25)$ & $119 / 433(27)$ & $0.90(0.65-1.24)$ & \\
\hline \multicolumn{5}{|l|}{ Atopic } \\
\hline Yes & $113 / 458(25)$ & $138 / 481(29)$ & $0.83(0.61-1.13)$ & \multirow{2}{*}{0.391} \\
\hline No & $14 / 47(30)$ & $13 / 31(42)$ & $0.53(0.20-1.41)$ & \\
\hline \multicolumn{5}{|l|}{ Ethnic group } \\
\hline White & $34 / 148(23)$ & $31 / 164(17)$ & $1.28(0.70-2.33)$ & \multirow[t]{2}{*}{0.177} \\
\hline Non-white & $86 / 270(32)$ & $97 / 254(38)$ & $0.78(0.54-1.14)$ & \\
\hline \multicolumn{5}{|c|}{$\begin{array}{l}\text { Data are presented as } \mathrm{n} / \mathrm{N}(\%) \text { or OR }(95 \% \mathrm{CI}) \text {, unless otherwise stated. LTRA: leukotriene receptor } \\
\text { antagonist; ICS: inhaled corticosteroids; BUD: budesonide equivalent. \#: adjusted for randomised } \\
\text { controlled trial population, age and lexcept the analysis for higher versus lower ICS dose) dose of ICS } \\
\text { (budesonide equivalent); }{ }^{\uparrow} \text { : defined by International Obesity Task Force criteria. }\end{array}$} \\
\hline
\end{tabular}

in mean \pm SD age between those who did and did not lose control (12.8 \pm 3.0 and $12.6 \pm 2.9$ years, respectively) and no difference in baseline ICS dose (median (IQR) 400 (400-1000) $\mu \mathrm{g}$ for both those who did and did not lose control). The interaction terms between treatment arm and the five baseline participant characteristics for loss of asthma control were nonsignificant (supplementary table S3).

TABLE 4 Results from Cox regression models ${ }^{\#}$ analysing time to first exacerbation for subgroups of participants

\begin{tabular}{|c|c|c|c|}
\hline & \multicolumn{2}{|c|}{$\begin{array}{l}\text { Time to first exacerbation for participants } \\
\text { where treatment was guided by } F_{\mathrm{ENO}} \\
\text { compared to standard care }\end{array}$} & \multirow[t]{2}{*}{$\begin{array}{c}\text { Interaction } \\
\text { p-value }\end{array}$} \\
\hline & HR (95\% CI) & p-value & \\
\hline \multicolumn{4}{|l|}{ LTRA treatment } \\
\hline Yes & $0.76(0.57-0.99)$ & 0.048 & \multirow[t]{2}{*}{0.049} \\
\hline No & $1.26(0.82-1.90)$ & 0.292 & \\
\hline \multicolumn{4}{|l|}{ ICS dose } \\
\hline$\leqslant 400 \mu \mathrm{g}$ BUD & $0.76(0.52-1.12)$ & 0.166 & \multirow[t]{2}{*}{0.393} \\
\hline$>400 \mu \mathrm{g}$ BUD & $0.94(0.71-1.25)$ & 0.667 & \\
\hline \multicolumn{4}{|l|}{ Obese } \\
\hline Yes & $0.96(0.74-1.25)$ & 0.787 & \multirow[t]{2}{*}{0.456} \\
\hline No & $0.78(0.48-1.27)$ & 0.321 & \\
\hline \multicolumn{4}{|l|}{ Atopic } \\
\hline Yes & $0.61(0.29-1.31)$ & 0.207 & \multirow{2}{*}{0.347} \\
\hline No & $0.90(0.70-1.16)$ & 0.412 & \\
\hline \multicolumn{4}{|l|}{ Ethnic group } \\
\hline White & $1.24(0.76-2.02)$ & 0.391 & \multirow[t]{2}{*}{0.268} \\
\hline Non-white & $0.90(0.67-1.20)$ & 0.469 & \\
\hline $\begin{array}{l}F_{\text {ENO: }} \text { exhaled } n \\
\text { corticosteroids; } \\
\text { group + subgrou } \\
\text { where outcomes }\end{array}$ & $\begin{array}{l}\text {; HR: hazard rati } \\
\text { equivalent. \#: the } \\
\text { ge + study ID + ba } \\
\text { groups were analy }\end{array}$ & $\begin{array}{l}\text { ene recep } \\
\text { itted as ti } \\
\text { ine ICS Wo }\end{array}$ & $\begin{array}{l}\text { ICS: inhaled } \\
+ \text { treatment } \\
\text { in the model }\end{array}$ \\
\hline
\end{tabular}



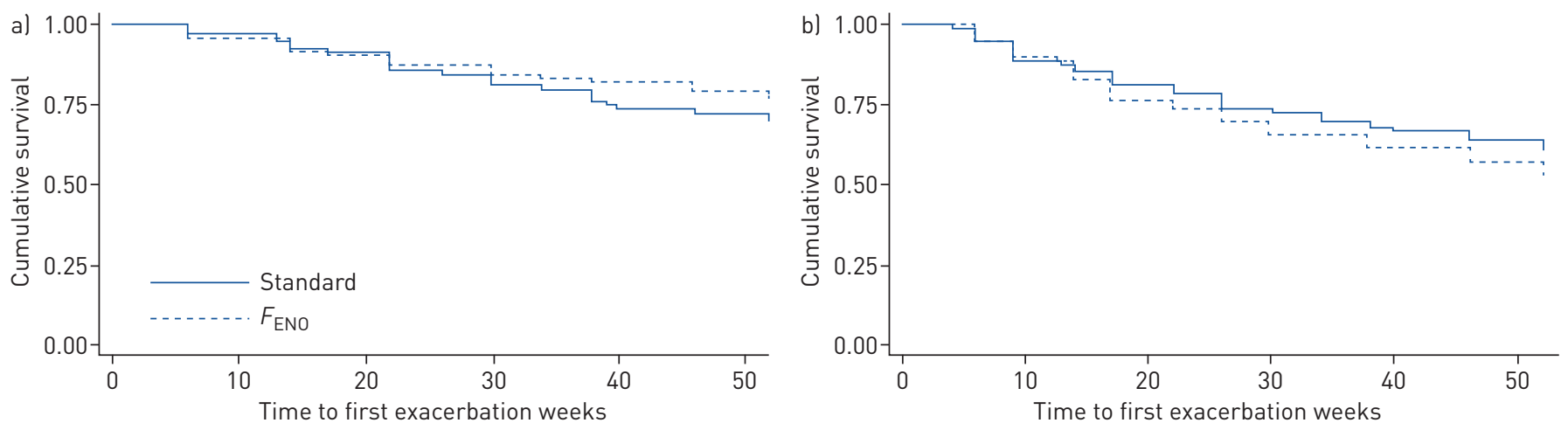

FIGURE 1 Kaplan-Meier curves showing time to first exacerbation for patients whose asthma treatment was guided by either exhaled nitric oxide fraction ( $F_{E N O}$ ) or by symptoms only (standard) and stratified by leukotriene receptor antagonist (LTRA) treatment. a) Patients not treated with LTRA; b) patients treated with LTRA. The difference between treatment arms was significant for those not treated with LTRA ( $p=0.048$ ), but not for the patients treated with LTRA ( $p=0.292$ ).

However, there was an indication of reduced odds of loss of control in the $F_{\text {ENO }}$ arm versus standard arm in those subgroups of participants who were not on LTRA at baseline, and in those who were not obese at baseline (supplementary table S3). The number of controlled participants needed to treat with $F_{\text {ENO }}$ guided management to prevent one losing control among those not treated with LTRA was 11.

\section{Time to loss of control}

Within the subgroup who lost control $(n=344)$ the median (IQR) time to loss of control was 17 (13-30) weeks with standard treatment and 22 (13-34) weeks with $F_{\mathrm{ENO}}$-guided treatment. The interaction terms with treatment arm were not significant for ICS dose $\leqslant 400 \mu \mathrm{g}$ versus $>400 \mu \mathrm{g}$, atopy, LTRA treatment, white versus other race or obesity (yes or no) (table 5). There was borderline evidence of a longer time to first loss of control for $F_{\mathrm{ENO}}$-guided compared to standard treatment within subgroups who were not treated with LTRA (HR 0.77, 95\% CI 0.60-0.99) (figure 2), nonobese (HR 0.77, 95\% CI 0.61-0.99) (figure 3) and atopic (HR $0.80,95 \%$ CI $0.63-1.00$ ) (table 5 and supplementary figure $\mathrm{S} 1$ ).

TABLE 5 Results from Cox regression models ${ }^{\#}$ analysing time to first loss of control for subgroups of participants, all of whom were controlled at baseline

\section{Time to first exacerbation for participants where treatment was guided by $F_{\mathrm{ENO}}$ compared to standard care}

\begin{tabular}{|c|c|c|c|}
\hline & & \\
\hline & HR $(95 \% \mathrm{CI})$ & p-value & \\
\hline \multicolumn{4}{|l|}{ LTRA treatment } \\
\hline Yes & $0.77(0.60-0.99)$ & 0.038 & 0.230 \\
\hline No & $1.05(0.68-1.64)$ & 0.822 & \\
\hline \multicolumn{4}{|l|}{ ICS dose } \\
\hline$\leqslant 400 \mu \mathrm{g}$ BUD & $0.82(0.62-1.10)$ & 0.182 & 0.899 \\
\hline$>400 \mu \mathrm{g}$ BUD & $0.84(0.62-1.16)$ & 0.293 & \\
\hline \multicolumn{4}{|l|}{ Obese } \\
\hline Yes & $0.77(0.61-0.99)$ & 0.042 & 0.130 \\
\hline No & $1.15(0.73-1.81)$ & 0.538 & \\
\hline \multicolumn{4}{|l|}{ Atopic } \\
\hline Yes & $1.29(0.54-3.08)$ & 0.566 & 0.293 \\
\hline No & $0.80(0.63-1.00)$ & 0.050 & \\
\hline \multicolumn{4}{|l|}{ Ethnic group } \\
\hline White & $0.85(0.58-1.24)$ & 0.396 & 0.970 \\
\hline Non-white & $0.85(0.64-1.14)$ & 0.289 & \\
\hline
\end{tabular}



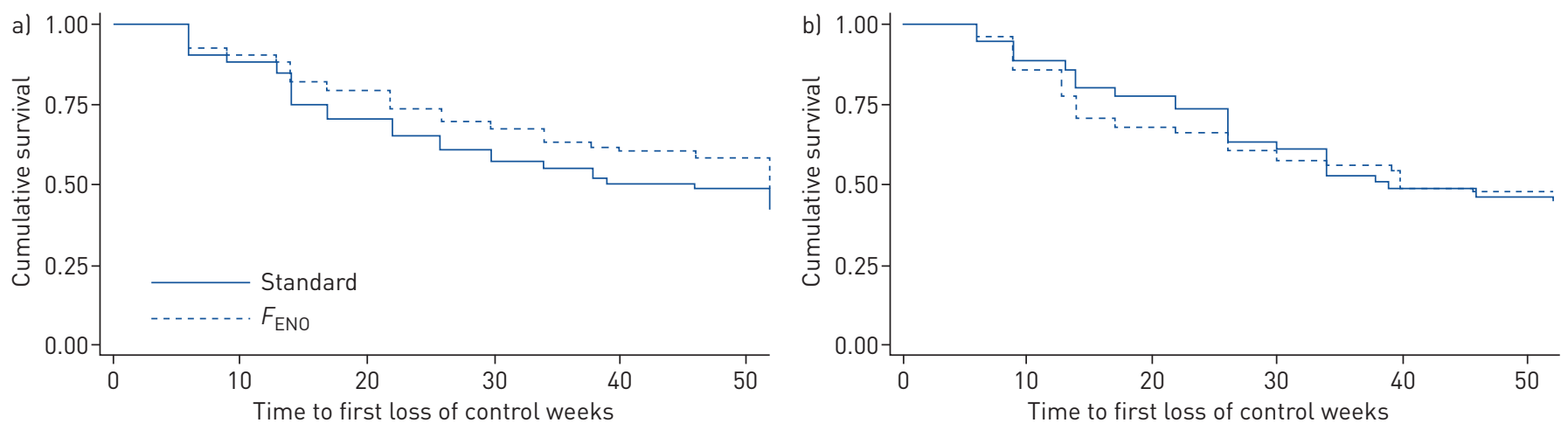

FIGURE 2 Kaplan-Meier curves showing time to loss of control for patients who were initially controlled and whose asthma treatment was guided by either exhaled nitric oxide fraction ( $F_{\mathrm{ENO}}$ ) or by symptoms only (standard) and stratified by leukotriene receptor antagonist (LTRA) treatment. a) Patients not treated with LTRA; b) patients treated with LTRA. The difference between treatment arms was significant for those not treated with LTRA ( $p=0.038$ ), but not for the patients treated with LTRA ( $p=0.822$ ).
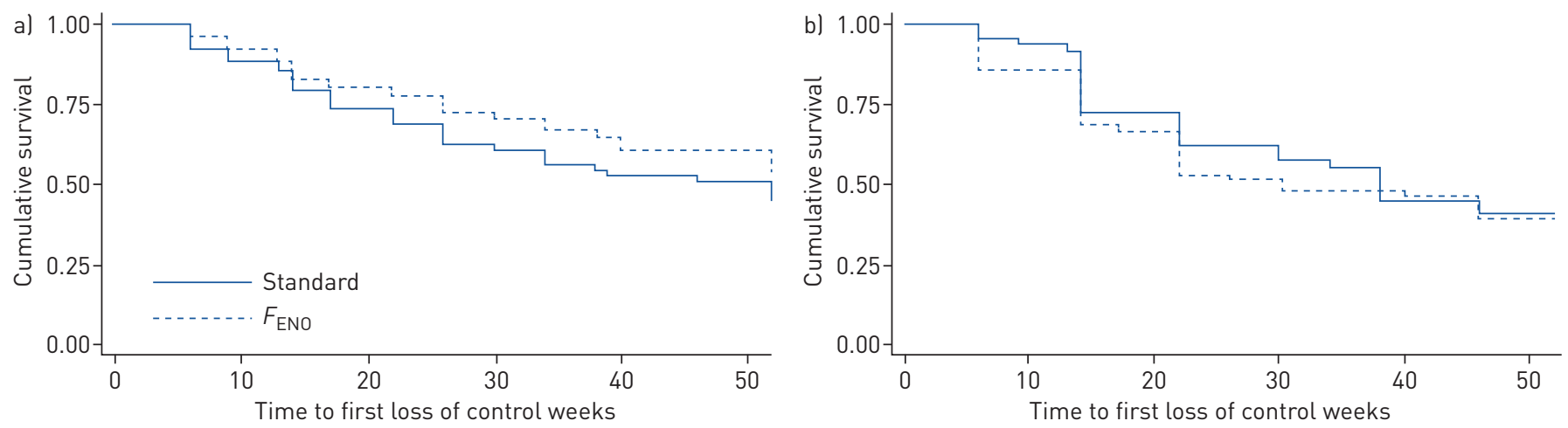

FIGURE 3 Kaplan-Meier curves showing time to loss of control for patients who were initially controlled and whose asthma treatment was guided by either exhaled nitric oxide fraction $\left(F_{\text {ENO }}\right)$ or by symptoms only (standard) and stratified by obesity status. a) Nonobese patients; b) obese patients. The difference between treatment arms was significant for those who were not obese ( $p=0.042)$, but not for the patients who were obese $(p=0.538)$.

\section{Discussion}

We analysed data collected in seven RCTs to test the hypothesis that there are subgroups of patients in whom $F_{\mathrm{ENO}}$-guided treatment is more effective in improving asthma outcomes compared to standard treatment. The main finding was that within these RCTs, the odds for exacerbation and loss of control for those not treated with LTRA were $32 \%$ and $30 \%$ lower, respectively, in the $F_{\mathrm{ENO}}$-guided arm compared to standard treatment. The significant interaction term for LTRA treatment and treatment for exacerbation indicated that $F_{\mathrm{ENO}}$-driven management may have reduced exacerbations for those not treated with LTRA, but not among those treated with LTRA. A second finding was that outcomes were no different between groups stratified by ICS dose or ethnic group. Collectively, these findings support the hypothesis that $F_{\mathrm{ENO}}$ is more useful for guiding treatment compared to standard practice in children with asthma not treated with LTRA.

A further finding was that in nonobese participants (but not in obese participants), $F_{\mathrm{ENO}}$-guided treatment was associated with a $31 \%$ reduction in odds for loss of control compared to standard treatment and when control was lost, time to loss of control was longer. Although the interaction term for obesity and treatment for loss of control was not significant, we believe that the improved outcomes for nonobese children merits further consideration. There was consistency in our results (i.e. an association with any loss of control and time to loss of control) and also there is biological plausibility whereby asthma associated with obesity may be a separate non-eosinophilic phenotype, especially in females [33]. A systematic review found no evidence of increased or reduced asthma control among children who were obese [34], and asthma guidelines do not recommend different treatment approaches for obese patients with asthma [4-6]. Further research is required to clarify whether $F_{\text {ENO-guided treatment is equally }}$ effective in obese and nonobese children. 
Our observation that time to loss of control was longer among children who were atopic receiving

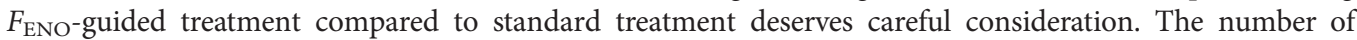
nonatopic participants included in our analysis was relatively small, since atopy was an inclusion criterion for four cohorts $[18,19,21,23]$ and the atopic subgroup were no more or less likely to have an exacerbation or to lose control within the trials. Since $F_{\mathrm{ENO}}$ is considered to be a surrogate for allergic or eosinophilic airway inflammation [35], it is biologically plausible that $F_{\mathrm{ENO}}$-guided treatment algorithms are more likely to suppress airway inflammation and improve asthma control. Further evidence of biological plausibility comes from an RCT whose data are included in our analysis [14], which found fewer days with maximal symptoms among those with elevated IgE and multiple positive skin-prick tests. Although nonatopic asthma is less common than atopic asthma, e.g. present in $18 \%$ of participants in the three trials which did not include only atopic participants [14, 20, 22], asthma is a very common condition and there are $\sim 150000-200000$ nonatopic asthmatic children in the UK [1]. There is a need to establish whether treatment and monitoring for atopic and nonatopic children should be the same.

The magnitude of significantly reduced risk for exacerbations and loss of control in the intervention compared to standard treatment was typically $25-30 \%$, and this difference is clinically meaningful since it is consistent with the benefit seen from commonly used asthma treatments such as LTRA and ICS. KNORR et al. [36] report a $23 \%$ reduced incidence of exacerbations in young children treated with montelukast compared to placebo. The review by CALPIN et al. [37] reports a $32 \%$ reduced risk for oral steroid treatment for exacerbations among children treated with ICS compared to placebo.

The RCTs included in our study applied different inclusion criteria, $F_{\mathrm{ENO}}$-guided treatment algorithms and asthma control scores, and these methodological differences will weaken any relationship between the intervention and asthma outcomes. The seven RCTs did apply a standard definition of exacerbation and apparatus for measuring $F_{\mathrm{ENO}}$. Despite the differences between RCTs, we still observed differences in outcomes between some of the subgroups studied, and it is likely that the magnitude of difference that we report in outcomes between the subgroups stratified by LTRA treatment, obesity and atopy may be an underestimate of the true value.

Our study was not designed to determine why $F_{\mathrm{ENO}}$-guided treatment was associated with improved asthma outcomes among those not treated with LTRA compared to participants receiving LTRA treatment. Treatment with LTRA is known to reduce $F_{\mathrm{ENO}}$ by $\sim 25 \%$ in children with atopic asthma [31] and may plausibly confound $F_{\mathrm{ENO}}$-guided treatment, especially since the RCT treatment algorithms did

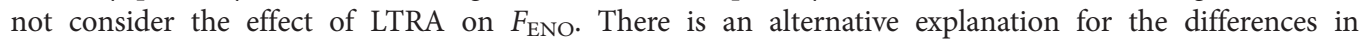
exacerbation outcomes associated with LTRA treatment in different RCT arms; those treated with LTRA were younger and had more severe asthma (including higher ICS dose, needing LABA treatment and almost twice the exacerbation prevalence) and $F_{\mathrm{ENO}}$-guided asthma treatment may be less effective in more severe asthma rather than in children receiving LTRA treatment per se. Given that LTRA are commonly used in asthma treatment, there is a need to study the impact of LTRA treatment on $F_{\mathrm{ENO}}$-guided asthma treatment.

We observed that when data from the RCTs were combined, $F_{\mathrm{ENO}}$-guided asthma treatment was associated with reduced risk for loss of control and time to loss of control among nonobese children. This contrasts with the findings of an RCT whose data are included in the present analysis [14], which reported fewer symptoms among obese participants (i.e. BMI $>30 \mathrm{~kg} \cdot \mathrm{m}^{-2}$ ) receiving $F_{\mathrm{ENO}}$-guided treatment. This apparent inconsistency may be due to several factors. First, the outcome in the study by SzEFLER et al. [14] was days of maximal symptoms, but this variable was not available in all the RCTs included in the present article and therefore loss of control was the outcome analysed here. Second, participants were all of African American or Hispanic ethnic origin, on higher ICS dose and had a considerably higher obesity prevalence [14], and some or all of these difference characteristics could explain different outcomes compared to the remaining six RCT participants. In our study, the reduced odds for loss of control and time to loss of control for nonobese children receiving $F_{\mathrm{ENO}}$-guided treatment compared to standard treatment is likely to be underestimated due to inclusion of $F_{\mathrm{ENO}}$ and asthma control data from the RCT of SZEFLER et al. [14].

There are some limitations to our study. First, the time to loss of control or first exacerbation was restricted to the predetermined assessment periods and this lack of precision will weaken the reported differences in these outcomes between subgroups. Second, the RCTs had different study designs with different step-up/step-down criteria and management regimes. Third, ethnicity data was only available for four of the seven RCTs and was therefore not included as a covariate in the models, but ideally we would have included ethnicity in our model since ethnicity was associated with differences between the other subgroups analysed (supplementary table S2). Fourth, ideally we would have performed a sensitivity analysis by excluding participants in the RCT by SzEFLER et al. [14], since their characteristics were 
different to the remaining RCTs for age, ethnicity and obesity, but this would have resulted in a $50 \%$ smaller sample size and the analysis would have been underpowered. A final limitation is that self-reported ICS adherence was available in only three RCTs included in our study [14, 22, 23], so we were not able to compare outcomes between treatment arms between adherent and nonadherent participants. Future research could test the hypothesis that asthma outcomes are improved by $F_{\mathrm{ENO}}$-guided treatment in adherent compared to nonadherent patients.

In summary, we have used data from $>1000$ asthmatic children and report that $F_{\mathrm{ENO}}$-guided treatment lead to better asthma outcomes among those not treated with LTRA.

Data availability: Data used in this study are available on request to the data custodians of the individual trials.

Conflict of interest: S. Fielding has nothing to disclose. M. Pijnenburg has nothing to disclose. J. de Jongste has nothing to disclose. K. Pike has nothing to disclose. G. Roberts has nothing to disclose. H. Petsky has nothing to disclose. A.B. Chang reports grants (project, Centre for Research Excellence and practitioner fellowship) from the National Health and Medical Research Council, Australia during the conduct of the study; and other potential conflict from being an author in UpToDate and USA Chest Chronic Cough Guidelines outside the submitted work. M. Fritsch has nothing to disclose. T. Frischer has nothing to disclose. S.J. Szefler has nothing to disclose. P. Gergen has nothing to disclose. F. Vermeulen has nothing to disclose. R. Vael has nothing to disclose. S.S. Turner has nothing to disclose.

\section{References}

1 Asthma UK. Asthma Facts and FAQs. www.asthma.org.uk/asthma-facts-and-statistics Date last accessed: August 31, 2017.

2 Centers for Disease Control and Prevention. Asthma: Most Recent Data. www.cdc.gov/asthma/most_recent_data. htm 2016 Date last accessed: August 31, 2017. Date last updated: March 25, 2019.

3 World Health Organization. Asthma. www.who.int/respiratory/asthma/en/ Date last accessed: December 7, 2018.

4 Global Initiative for Asthma. Global Strategy for Asthma Management and Prevention. https://ginasthma.org/ gina-reports/ Date last accessed: July 26, 2019.

5 British Thoracic Society and Scottish Intercollegiate Guidelines Network. SIGN 158. The British Guideline on the Management of Asthma. www.brit-thoracic.org.uk/quality-improvement/guidelines/asthma/ Date last accessed: July 27, 2019.

6 National Institute for Health and Care Excellence. Asthma: Diagnosis, Monitoring and Chronic Asthma Management. www.nice.org.uk/guidance/ng80 Date last accessed: January 28, 2018.

7 Pavord ID, Beasley R, Agusti A, et al. After asthma: redefining airways diseases. Lancet 2018; 391: 350-400.

8 Pijnenburg MW, Hofhuis W, Hop WC, et al. Exhaled nitric oxide predicts asthma relapse in children with clinical asthma remission. Thorax 2005; 60: 215-218.

9 Zacharasiewicz A, Wilson N, Lex C, et al. Clinical use of noninvasive measurements of airway inflammation in steroid reduction in children. Am J Respir Crit Care Med 2005; 171: 1077-1082.

10 Beck-Ripp J, Griese M, Arenz S, et al. Changes of exhaled nitric oxide during steroid treatment of childhood asthma. Eur Respir J 2002; 19: 1015-1019.

11 Baraldi E, Azzolin NM, Zanconato S, et al. Corticosteroids decrease exhaled nitric oxide in children with acute asthma. J Pediatr 1997; 131: 381-385.

12 Silkoff PE, Carlson M, Bourke T, et al. The Aerocrine exhaled nitric oxide monitoring system NIOX is cleared by the US Food and Drug Administration for monitoring therapy in asthma. J Allergy Clin Immunol 2004; 114: 1241-1256.

13 Petsky HL, Kew KM, Chang AB. Exhaled nitric oxide levels to guide treatment for children with asthma. Cochrane Database Syst Rev 2016; 11: CD011439.

14 Szefler SJ, Mitchell H, Sorkness CA, et al. Management of asthma based on exhaled nitric oxide in addition to guideline-based treatment for inner-city adolescents and young adults: a randomised controlled trial. Lancet 2008; 372: 1065-1072.

15 Voorend-van Bergen S, Vaessen-Verberne AA, Landstra AM, et al. Fractional exhaled nitric oxide monitoring does not improve asthma management in children with concordant and discordant asthma phenotypes. Am J Respir Crit Care Med 2015; 192: 1016-1018.

16 Fielding S, Pijnenburg M, de Jongste JC, et al. Change in $\mathrm{FEV}_{1}$ and $F_{\mathrm{ENO}}$ measurements as predictors of future asthma outcomes in children. Chest 2019; 155: 331-341.

17 Turner S. Exhaled nitric oxide and the management of childhood asthma - yet another promising biomarker "has been" or a misunderstood gem. Paediatr Respir Rev 2015; 16: 88-96.

18 Fritsch M, Uxa S, Horak FJ, et al. Exhaled nitric oxide in the management of childhood asthma: a prospective 6-months study. Pediatr Pulmonol 2006; 41: 855-862.

19 Peirsman EJ, Carvelli TJ, Hage PY, et al. Exhaled nitric oxide in childhood allergic asthma management: a randomised controlled trial. Pediatr Pulmonol 2014; 49: 624-631.

20 Petsky HL, Li AM, Au CT, et al. Management based on exhaled nitric oxide levels adjusted for atopy reduces asthma exacerbations in children: a dual centre randomized controlled trial. Pediatr Pulmonol 2015; 50: 535-543.

21 Pijnenburg MW, Bakker EM, Hop WC, et al. Titrating steroids on exhaled nitric oxide in children with asthma: a randomized controlled trial. Am J Respir Crit Care Med 2005; 172: 831-836.

22 Pike K, Selby A, Price S, et al. Exhaled nitric oxide monitoring does not reduce exacerbation frequency or inhaled corticosteroid dose in paediatric asthma: a randomised controlled trial. Clin Respir J 2013; 7: 204-213.

23 Voorend-van Bergen S, Vaessen-Verberne AA, Brackel HJ, et al. Monitoring strategies in children with asthma: a randomised controlled trial. Thorax 2015; 70: 543-550.

24 Schatz M, Sorkness CA, Li JT, et al. Asthma Control Test: reliability, validity, and responsiveness in patients not previously followed by asthma specialists. J Allergy Clin Immunol 2006; 117: 549-556. 
25 Santanello NC, Barber BL, Reiss TF, et al. Measurement characteristics of two asthma symptom diary scales for use in clinical trials. Eur Respir J 1997; 10: 646-651.

26 Verberne AA, Frost C, Roorda RJ, et al. One year treatment with salmeterol compared with beclomethasone in children with asthma. The Dutch Paediatric Asthma Study Group. Am J Respir Crit Care Med 1997; 156: 688-695.

27 Wasserfallen JB, Gold K, Schulman KA, et al. Development and validation of a rhinoconjunctivitis and asthma symptom score for use as an outcome measure in clinical trials. J Allergy Clin Immunol 1997; 100: 16-22.

28 Cole TJ, Bellizzi MC, Flegal KM, et al. Establishing a standard definition for child overweight and obesity worldwide: international survey. BMJ 2000; 320: 1240-1243.

29 Quanjer PH, Stanojevic S, Cole TJ, et al. Multi-ethnic reference values for spirometry for the 3-95-yr age range: the global lung function 2012 equations. Eur Respir J 2012; 40: 1324-1343.

30 American Thoracic Society, European Respiratory Society. ATS/ERS recommendations for standardized procedures for the online and offline measurement of exhaled lower respiratory nitric oxide and nasal nitric oxide, 2005. Am J Respir Crit Care Med 2005; 171: 912-930.

31 Montuschi P, Mondino C, Koch P, et al. Effects of a leukotriene receptor antagonist on exhaled leukotriene E4 and prostanoids in children with asthma. J Allergy Clin Immunol 2006; 118: 347-353.

32 Koo S, Gupta A, Fainardi V, et al. Ethnic variation in response to IM triamcinolone in children with severe therapy-resistant asthma. Chest 2016; 149: 98-105.

33 Jensen ME, Gibson PG, Collins CE, et al. Airway and systemic inflammation in obese children with asthma. Eur Respir J 2013; 42: 1012-1019.

34 Ahmadizar F, Vijverberg SJH, Arets HGM, et al. Childhood obesity in relation to poor asthma control and exacerbation: a meta-analysis. Eur Respir J 2016; 48: 1063-1073.

35 Payne DN, Adcock IM, Wilson NM, et al. Relationship between exhaled nitric oxide and mucosal eosinophilic inflammation in children with difficult asthma, after treatment with oral prednisolone. Am J Respir Crit Care Med 2001; 164: 1376-1381.

36 Knorr B, Franchi LM, Bisgaard H, et al. Montelukast, a leukotriene receptor antagonist, for the treatment of persistent asthma in children aged 2 to 5 years. Pediatrics 2001; 108: E48.

37 Calpin C, Macarthur C, Stephens D, et al. Effectiveness of prophylactic inhaled steroids in childhood asthma: a systemic review of the literature. J Allergy Clin Immunol 1997; 100: 452-457. 